\title{
Prediction of Bearing Remaining Useful Life Based on Euclidean Distance Using an Artificial Neural Network Approach
}

\author{
Dalia M. Ammar, Mohammad A. Younes and Elsayed S. Elsayed \\ Department of Production Engineering, Faculty of Engineering, Alexandria University \\ Alexandria, EGYPT
}

\begin{abstract}
Accurate prediction of Remaining Useful Life (RUL) of machines and machine components is very important for reliability evaluation. This paper proposes an Artificial Neural Network (ANN) as a method for accurate prediction of RUL of bearings based on vibration measurements during an accelerated life test. The input features to the neuro-predictor are: the vibration signal in the time domain, the dominant harmonics of the bearing vibration signal expressed in a set of selected coefficients of the discrete cosine transforms (DCT), and the main harmonics of the vibration signal as expressed by Fast Fourier Transform (FFT). The Euclidean distance which is a measure of time to failure based on RMS value is used as the figure of merit for the validation of the ANN. Henceforth; the RUL of the bearing can be predicted as the output of the neuropredictor. The results prove that the suggested methodology can successfully be applied for prediction of bearing RUL.
\end{abstract}

Keywords - Remaining Useful Life (RUL), Reliability evaluation, Artificial Neural Network (ANN), Discrete Cosine Transform (DCT), Machine Tool Dynamics.

\section{Introduction}

Failures occurring during production processes result in negative implications. To overcome these implications; several methods to detect machinery faults have evolved to safety. Analysis of reliability data plays an important role in the maintenance decision making process [1]. Existing methods for predicting machine element failures can be grouped into two main categories: traditional and prognostics reliability estimation approaches [2].

Traditional approaches are based on the distribution of event records of a population of identical units. Many parametric failure models, such as Poisson, Exponential, Weibull and Log-Normal distributions have been used to model machine reliability [3]. The most popular among them is the Weibull distribution due to its versatility and ability to accommodate various types of behavior including infant mortality, regular performance and wear out phases [3]. On the other hand, prognostic approaches aim to predict how much time is left before a failure occurs given the current machine condition and past operation record. While diagnosis involves identifying and quantifying the damage that has already occurred, prognosis is concerned with predicting the damage that is yet to occur $[4,5]$. In fact, prognosis relies on diagnosis outputs [6].
The existing prognosis - or RUL prediction methods - can be classified into physical based prognosis models and data driven prognosis model [7]. Data driven prognosis models do not require assumptions of physical parameters or performance models thus, it is easy to apply. However, these techniques need a large amount of data to be as close to the real application as possible. Artificial Neural Networks (ANN) are well known as data driven techniques where historical data are used to train the neuron predictor to estimate a system performance parameter based on known input data which produce specific values of that performance parameter. The trained ANN will then be used to predict unknown performance parameters based on a given set of input data. Normally, ANNs consist of an input layer, one or several hidden layers and an output layer [8].

In this study a method is proposed to evaluate the RUL of deep groove ball bearings based on recorded vibration signals at different time intervals during an accelerated life test. In the proposed method, recorded vibration signals were used in three different ways as illustrated below in section 3 . The extracted features were used as input parameters to an ANN to predict bearing RUL. The proposed algorithm differs depending on the domain features and hence, training speed also differs. The performance of these different neural networks has been compared.

This paper is organized as follows. In section 2, the experimental setup is introduced. Then in section 3, applied methodology is developed. Section 4 presents results and discussion. The conclusions of this comparative study are summarized in sections 5 .

\section{Experimental work}

An experimental setup, Fig. 1, has been designed and built to carry out an accelerated life test of deep groove ball bearings. Two ball bearings were used to support a $25 \mathrm{~mm}$ diameter steel shaft. The shaft was driven by a variable speed induction motor $(380 \mathrm{~V} / 50 \mathrm{~Hz} / 1.5 \mathrm{HP} / 2$-poles/3500rpm) via a flexible coupling. The free end of the shaft was loaded through a lever arm pivoted on another ball bearing. The arm was used to magnify the load transmitted to the free end of the shaft and the load was applied on the free end of the arm. Fig. 2 shows a schematic diagram of the test rig. Five vibration signals were recorded for the three deep groove ball bearings. Vibration signals were recorded in the vertical and horizontal directions for each of the two bearings $\mathrm{R}$ and $\mathrm{F}$. For bearing $\mathrm{P}$, only 
vibration signal in the vertical direction was recorded. The deep groove ball bearings were manufactured by FSB [9]. All vibration data readings were recorded at appropriate intervals of time using B \& $\mathrm{K}$ accelerometers (Type 4381) having a voltage sensitivity equal to $8 \mathrm{mV} / \mathrm{ms}^{-2}$ and a frequency range up to $4800 \mathrm{HZ}$. Data collection was performed using a Brüel and Kjaer 4 Channel Pulse Analyzer (Type 2825) and Pulse Lap shop software (Version 6.1).

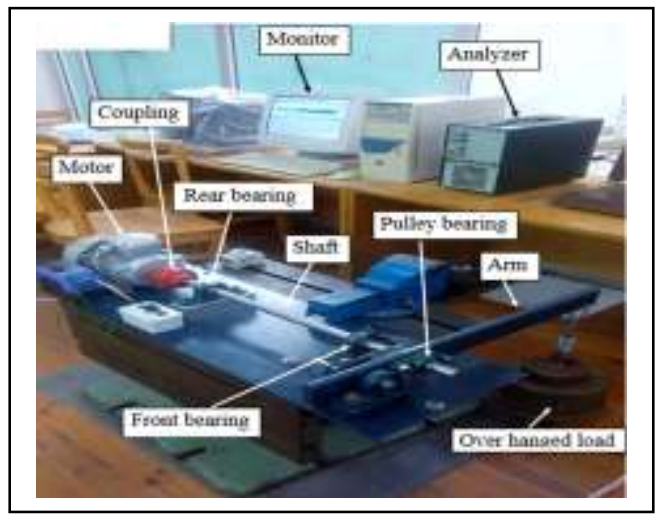

Figure1. Image of the experimental setup

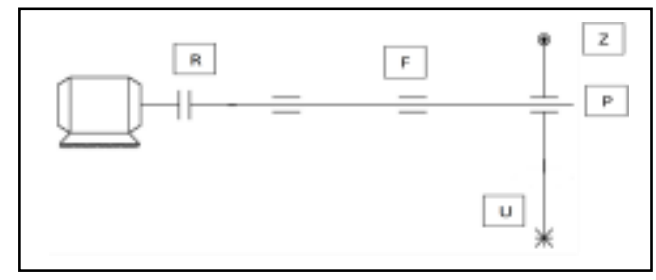

Figure 2-a. A schematic diagram of the test rig

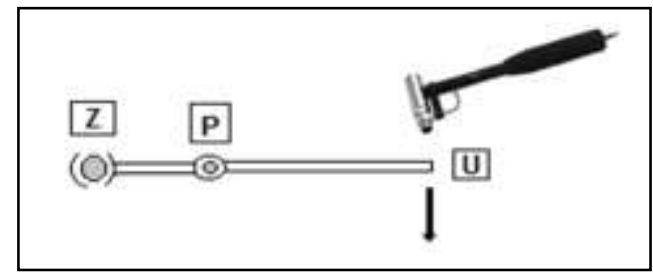

Figure 2-b. A schematic diagram of the modal test

To better understand and interpret the recorded results, an experimental modal test was performed to obtain the dynamic modal characteristics of the system at points R, F, and P. The test was performed by applying an excitation, using $\mathrm{B} \& \mathrm{~K}$ impact hammer (Type 8202) fitted with a B\&K force transducer (Type 8200) having an output sensitivity of 1.01 $\mathrm{pc} / \mathrm{N}$, Fig. 2-b, at the location of the applied force, point (U) and in the vertical direction to simulate the actual force. The response was recorded in both vertical and horizontal directions at front and rear bearings, points $F$ and $R$ respectively. For arm bearing at point $\mathrm{P}$, the response was measured in the vertical direction only.

\section{Methodology}

Fig. 3 shows a block diagram of the methodology followed in the present work.

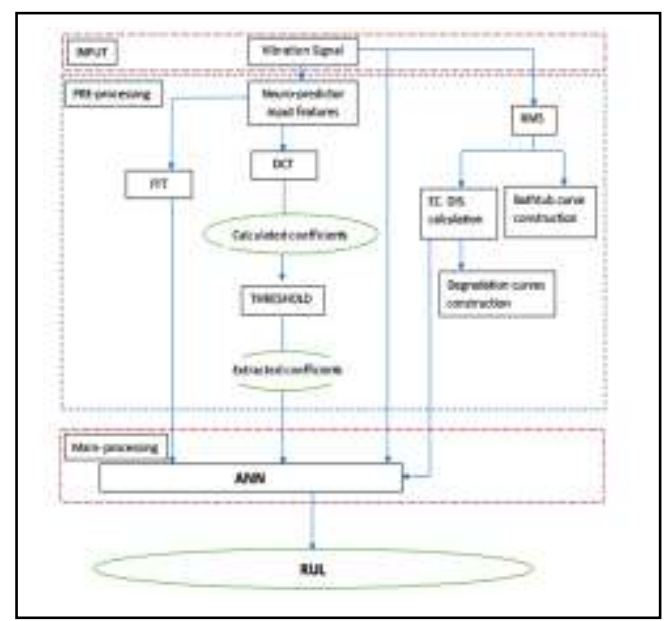

Figure 3. Block diagram for the bearing RUL prediction using ANN and DCT

In the pre-processing stage input vibration signals are used to calculate the RMS values from which the Euclidean distances are calculated and the bathtub curve is constructed as well. The degradation curves are then constructed using the estimated Euclidean distances. Also the vibration signal was transformed into different domains and results used as inputs to the ANN predictor of RUL. The input features to the neuro predictor as illustrated in the block diagram are the vibration signal values in time domain, selected coefficients of the discrete cosine transform (DCT) of the vibration signal, and the dominant harmonics of the FFT of the vibration signal. Using each of the three described input features; three computer programs have been designed under MATLAB (R14). The programs are used to build, train, and optimize the ANNs for the groups of inputs and also for testing the ANN performance in RUL prediction.

Vibration signal recorded for a bearing in the vertical or horizontal directions carries important information regarding its health condition and expected life time. The overall vibration signal expressed in RMS of vibration amplitude is a one measurement value commonly used for detection of bearing failure [10]. The RMS value usually used to construct the bathtub curve, Fig. 4 is a typical example of the failure stages of one of the tested bearings. According to vibration severity as per ISO 10816-1 class I for small machines, the unacceptable level range is $4.5-45.9 \mathrm{~mm} / \mathrm{sec}$ RMS. In the present study, the life of the bearing is considered to end when the vibration level of the vibration signal reaches $12 \mathrm{~mm} / \mathrm{sec}$.

The RMS values of the recorded vibration signals are used also to estimate the Euclidean Distance (ED) and construct a degradation curve for the considered bearing, Fig.5. In 
mathematics, ED is the distance between two points that simply can be measured with a ruler, and is given by the Pythagorean Theorem. Euclidean distance is related to Euclid's geometric principles [11]. In the present study, ED is calculated using Eq. (1).

$$
E D^{2}=\left(R M S_{t r}-R M S_{t}\right)^{2}+\left(t_{r}-t\right)^{2}
$$

Where:

$\mathrm{RMS}_{\mathrm{t}}$ : Root Mean Square value at time (t).

$\mathrm{RMS}_{\mathrm{tr}}$ : Root Mean Square value at failure time (tr).

The ED is used as a figure of Merit (FOM) for the degradation rate of the bearing and in the supervised training for the validation of the ANN. The degradation curve, shown in Fig. 5 is a very useful tool for understanding the failure behavior and reliability of the bearing and estimating bearing RUL.

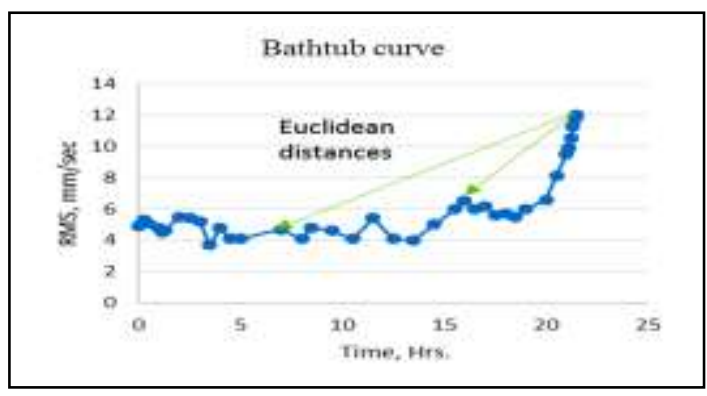

Figure 4. Bathtub curve and the Euclidean

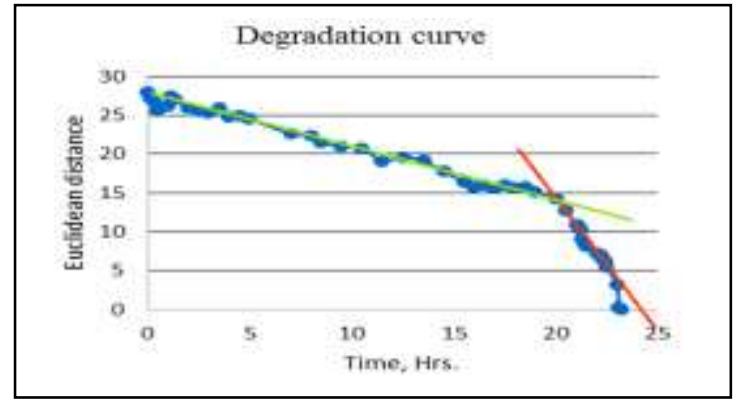

Figure 5. A typical degradation curve

\section{Results and discussion}

The degradation curve, Fig. 5, shows the change in the RUL with the service life of the bearing. Two distinct stages can be observed along the life span of the bearing with two different failure rates (change in the RUL). A constant rate of change in the RUL (constant failure rate) is observed in the early stage of the bearing life then a significant increase in the failure rate is observed near the end of the bearing life. As expected the first stage which represents the normal life of the bearing is much longer than the wear out stage near the end of the bearing life.

Fig. 6 shows two degradation curves of the front bearing $(F)$ based on the recorded vibration signals in the vertical direction, under two different conditions of the accelerated life test. The two life-tests were performed at same speed (3500 rpm) with two different loads (2288 $\mathrm{N}$ and $1987.5 \mathrm{~N})$. In the meantime, Fig. 7 shows two degradation curves of a similar bearing in the vertical direction at the same load of $2288 \mathrm{~N}$ but at two different speeds of $3500 \mathrm{rpm}$ and $1800 \mathrm{rpm}$.

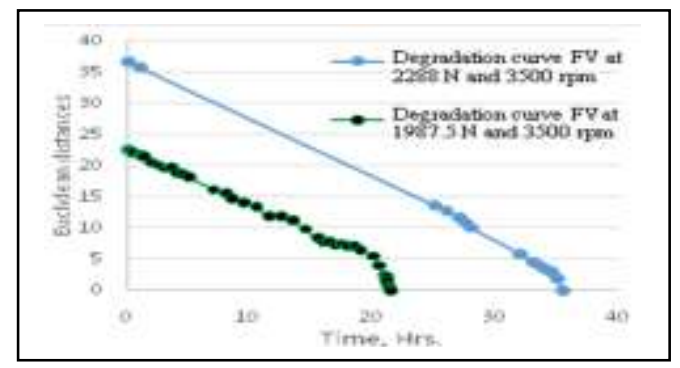

Figure 6. The degradation curves for FV at Loads $2288 \mathrm{~N}$ and $1987.5 \mathrm{~N}$, and the same speed $(3500 \mathrm{rpm})$

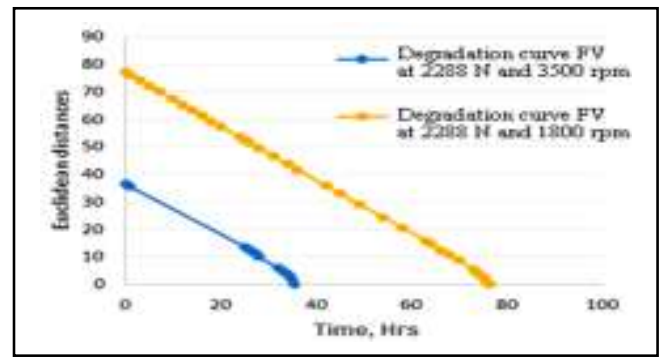

Figure 7. The degradation curves for FV at $3500 \mathrm{rpm}$ and $1800 \mathrm{rpm}$, and the same load $(2288 \mathrm{~N})$.

Fig. 6 shows the effect of the load on service life of a bearing at a high but constant speed. At $35000 \mathrm{rpm}$ and $2288 \mathrm{~N}$, the recorded bearing life was 35 hours. Decreasing the load to 1987.5 $\mathrm{N}$ led to a significant reduction in the bearing life to 22 hours. Although this results look unreasonable at first instant, however it can be explained by the fact that at speeds near the resonance of main system components lager loads seem to give a damping effect than relatively smaller loads. This justification is supported by results of the modal test represented by the frequency response function curve (FRF) of the test rig at loads of $2288 \mathrm{~N}$ and $1987.5 \mathrm{~N}$, Fig. 8. FRF represents the system output response due to an exciting force. In this test, FRF is measured in terms of $(\mathrm{mm} / \mathrm{sec}) / \mathrm{N}$. Analysis of Figs. 8-a and 8-b shows that the system has a resonance at frequency of $116 \mathrm{~Hz}$ (i.e. double the rotational speed $f_{r}$ of the system) and the magnitude of the FRF at that frequency is greater at a load of 2288 N, Fig. 8-b, compared with that at a load of 1987.5 N, Fig. 8 -a. This is also observed at most resonance frequencies above $200 \mathrm{~Hz}$, especially at bearing faults frequencies lying in this frequency range. This proves that the system at the lower load $1987.5 \mathrm{~N}$ is more sensitive to vibration and hence failed faster than that at the higher load $2288 \mathrm{~N}$.

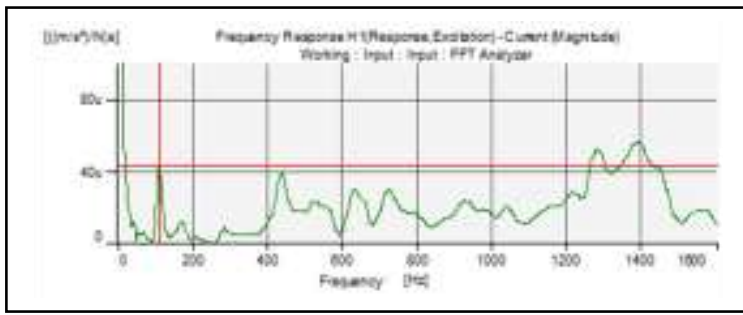


Proc. of the Fifth International Conference on Advances in Mechanical and Robotics Engineering - AMRE 2017. Copyright (C) Institute of Research Engineers and Doctors. All rights reserved. ISBN: 978-1-63248-123-8 doi: 10.15224/ 978-1-63248-123-8-15

Figure 8 -a. FRF curve at load $=2288 \mathrm{~N}$ and speed $=3500 \mathrm{rpm}$ for $\mathrm{FV}$

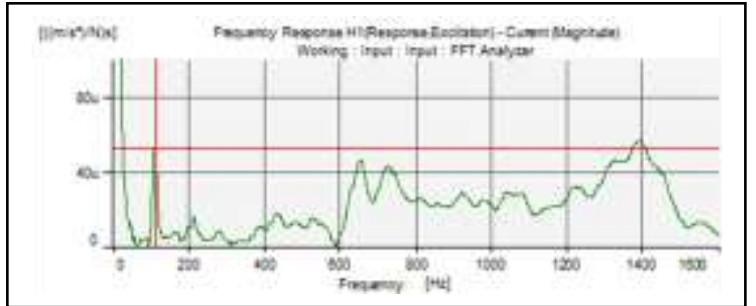

Figure 8-b. FRF curve at load=1987.5 $\mathrm{N}$ and speed $=3500 \mathrm{rpm}$ for $\mathrm{FV}$

The effect of speed on the service life of a bearing at a constant load is shown in Fig. 7. At 3500 rpm and $2288 \mathrm{~N}$, the recorded bearing life was 35 hours. Decreasing the speed to $1800 \mathrm{rpm}$ under the same load of $2288 \mathrm{~N}$ led to a significant increase in the bearing life to 80 hours. So, it can be concluded that running at higher speeds results in faster failure as long as the load remains the same.

Spectral analysis was carried out for the recorded vibration signals of the two life tests at two different loads, Fig. 6 and those at two different speeds, Fig.7, using the vibration analyzer to identify the generation and development of the bearing failure along its lifetime. Spectral analysis was carried out at three time intervals representing $5 \%, 60 \%$, and $92.5 \%$ of the bearing service life as illustrated in Figs. 9 and 10.

Fig. 9 shows the spectra of the front bearing vibration at a load of $2288 \mathrm{~N}$ and speed of $3500 \mathrm{rpm}$ after $3 \mathrm{hrs}$ of running (5\% of bearing life), Fig. 9 -a, after $25 \mathrm{hrs}$ of running ( $60 \%$ of bearing life), Fig. 9-b and after $33 \mathrm{hrs}$ of running (92\% of bearing life), Fig. 9-b. In the meantime, Fig. 10 shows the spectra at a load of $1987.5 \mathrm{~N}$ and speed of $3500 \mathrm{rpm}$ after $1.5 \mathrm{hrs}$ of running (5\% of bearing life), Fig. 10-a, after 14.5 hrs of running (60\% of bearing life), Fig. 10-b and after $20 \mathrm{hrs}$ of running (92\% of bearing life), Fig. 10-c.

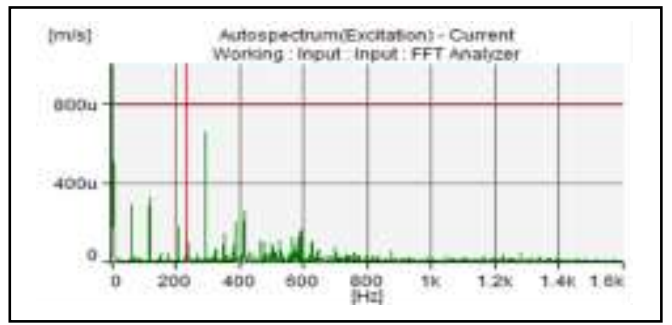

Figure 9-a. FFT spectrum of FV bearing vibration record at Load $=2288 \mathrm{~N}$ and Speed=3500 rpm after $1 \mathrm{hr}$ (5\% of the life span).

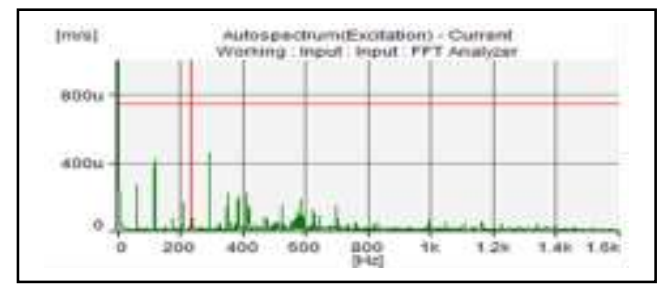

Figure 9-b. FFT spectrum of FV bearing vibration record at Load=2288 N and Speed=3500 rpm after $25 \mathrm{hrs}$ (after $60 \%$ of the life span).

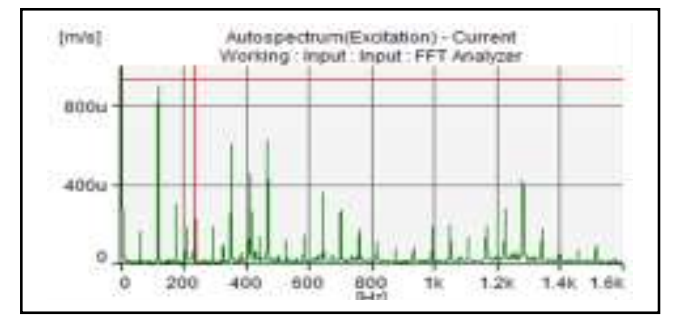

Figure 9-c. FFT spectrum of FV bearing vibration record at Load=2288 N and Speed $=3500 \mathrm{rpm}$ after $33 \mathrm{hrs}$ ( after $92 \%$ of the life span).

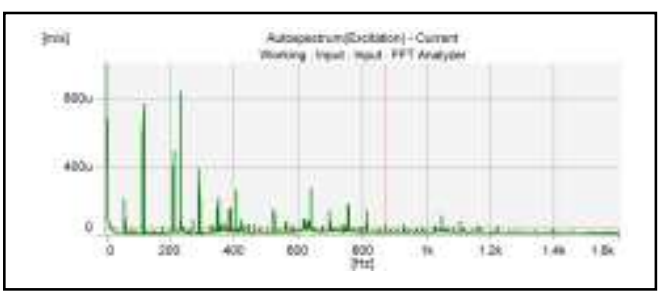

Figure 10-a. FFT spectrum of FV bearing vibration record at Load $=1987.5 \mathrm{~N}$ and Speed=3500 rpm after $1.15 \mathrm{hrs}$ (after $5 \%$ of the life span)

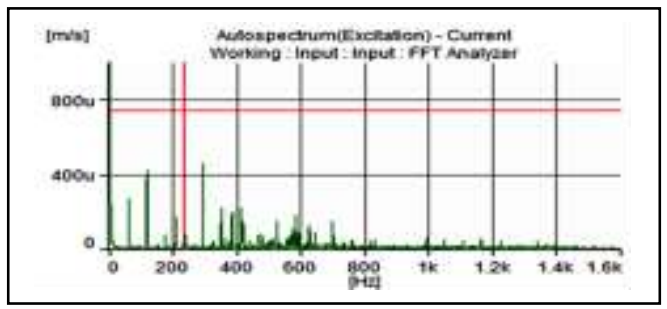

Figure 10-b. FFT spectrum of FV bearing vibration record at Load $=1987.5 \mathrm{~N}$ and Speed=3500 rpm after $14.5 \mathrm{hrs}$ (after $60 \%$ of the life span)

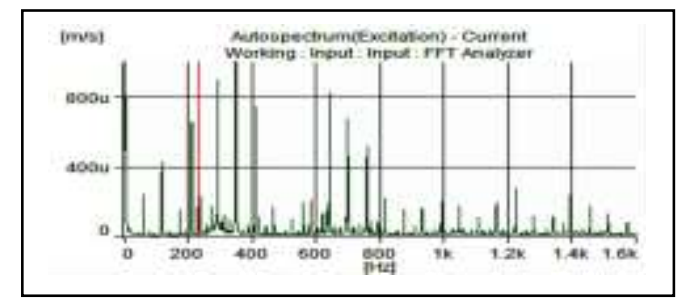

Figure 10-c. FFT spectrum of FV bearing vibration record at Load $=1987.5 \mathrm{~N}$ and speed $=3500 \mathrm{rpm}$ after $20 \mathrm{hrs}$ (after $92 \%$ of the life span)

Analysis of Figs. 9-a, 9-b and 9-c shows dominant harmonics at frequencies of 58 and $116 \mathrm{~Hz}$ which correspond to the rotational speed $\left(\mathrm{f}_{\mathrm{r}}\right)$ and its second harmonic. Also, dominant harmonics are observed at frequency $233 \mathrm{~Hz}$ which corresponds to the ball fault frequency (BSF) and also at its higher harmonics at 464, 697 and $930 \mathrm{~Hz}$. Near the end of the service life, Fig. 9-c, frequency components are observed at $640,1280 \mathrm{~Hz}$ which correspond to the second and fourth harmonics of the inner ring fault frequency (BPFI) and also side bands are observed at the first and second harmonics of rotational speed $(58$ and $116 \mathrm{~Hz}$ ) around the harmonics of both $\mathrm{BSF}$ and BPFI.

Examination of the three Figures shows significant increase in the amplitude value of the harmonics at $116 \mathrm{~Hz}(2 \mathrm{fr}), 232 \mathrm{~Hz}$ 
(BSF), $464 \mathrm{~Hz}$ (2 BSF), $697 \mathrm{~Hz}$ (3 BSF), and $930 \mathrm{~Hz}$ (4BSF) which indicates the failure of the balls of the bearing as running time increases. The increase in the value of the amplitude at $640 \mathrm{~Hz}(2 \mathrm{BPFI})$ and $1280 \mathrm{~Hz}(4 \mathrm{BPFI})$ indicates the failure of the inner ring of the bearing as running time increases. Inspection of Fig. 9 shows that up to $60 \%$ of the bearing service life, most of the dominant harmonics representing the bearing failure lie in the range up to $800 \mathrm{~Hz}$. Only few harmonics appear above this range near the end of the bearing life as seen at $92 \%$ of the service life, Fig. 9-c. Similar conclusions can also be drawn from Figs.10-a, 10-b, and 10-c, but the amplitudes are significantly higher and the rate of failure is much faster. Analysis of the FFT spectrum of the system vibration signal and its dominant harmonics provides an effective tool for diagnosis of bearing state of health which is an accurate indicator of its remaining useful life. A software module was developed for spectral analysis of the recorded vibration signals using both FFT and DCT. The developed software module can be used if the analyzer is not available.

Recorded vibration signals were transformed from the time domain to the frequency domain using DCT using the developed software. The DCT expresses a sequence of finite data in terms of a sum of cosine functions of different frequencies $[12,13]$. For a set of data $A=\left\{a_{0}, a_{1}, a_{2}, . ., a_{n-1}\right\}$, $a$ one dimensional discrete cosine transform coefficients can be computed using the following formula, Eq. (2):

$W(k)=\sum_{t=0}^{n=1} \alpha(t) \cdot \cos \left(\frac{(2 t+1) \pi k}{2 n}\right), \quad k=0,1, \ldots, n-1$

Where $\mathrm{n}$ is the number of data points.

Discrete cosine transform (DCT) coefficients are the weights that indicate how strong the frequency components are in the data. For each DCT coefficient, there is a base frequency $f$ and accordingly a base vector. If the set of data consists of correlated values, then most of the DCT coefficients will be either zero or very small numbers, and only a few are large.

Data dimensionality reduction is the main feature of DCT since most of the energy of the system is concentrated in a limited number of frequencies which will represent the selected DCT coefficients [14, 15]. Another software module was also developed under MATLAB (R14) to evaluate the DCT coefficients of the vibration signals. Calculated DCT coefficients for the considered cases are shown in Figs. 11 and 12, which show obvious change in the calculated DCT coefficients by the change in applied load, rotational speed and service life of tested bearings. Analysis of the DCT coefficients provides an effective tool for prediction of bearing state of health, consequently its remaining useful life.

Although FFT and DCT provide effective tools for prediction of bearing state of health and its remaining useful life, however correct interpretations need an expert with extensive knowledge and past experience. Artificial Neural Networks (ANN) as an artificial intelligence tool [16] provides a powerful means for prediction of bearings RUL based on the current state of the bearing as expressed in its vibration signal and the past records of its performance along its service life.

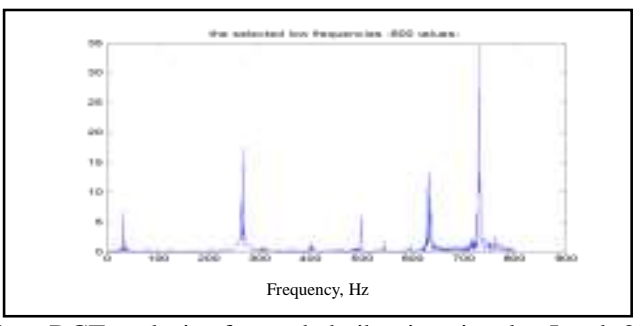

Figure 11-a. DCT analysis of recorded vibration signal at Load=2288 N and Speed $=3500 \mathrm{rpm}$, after $1 \mathrm{hr}$ ( $5 \%$ of the life span)

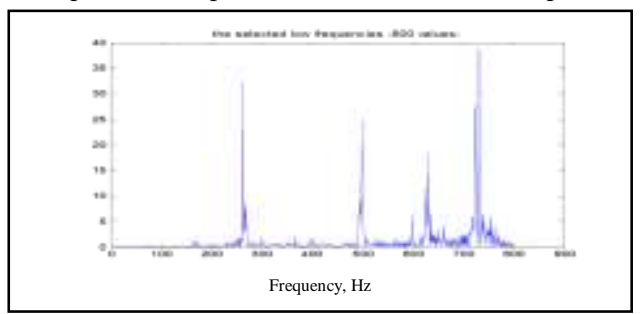

Figure 11-b. DCT analysis of recorded vibration signal at Load $=2288 \mathrm{~N}$ and Speed $=3500 \mathrm{rpm}$ after $25 \mathrm{hrs}$ (after $60 \%$ of the life span)

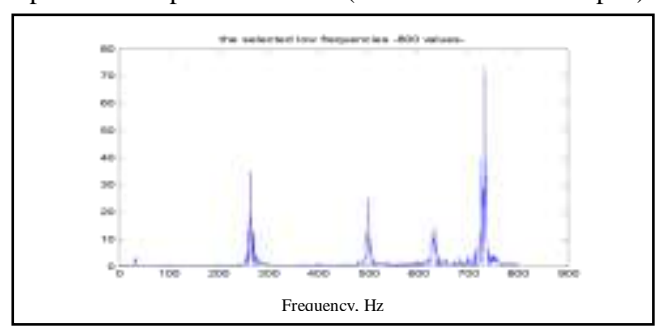

Figure 11-c. DCT analysis of recorded vibration signal at Load=2288 N and Speed=3500 rpm after $33 \mathrm{hrs}$ (after $92 \%$ of the life span)

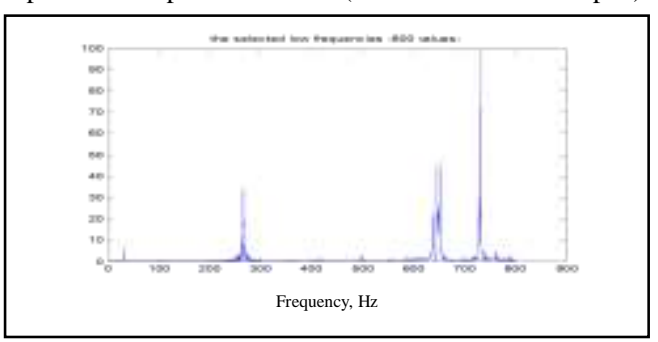

Figure 12-a. DCT analysis of recorded vibration signal at $\operatorname{Load}=1987.5 \mathrm{~N}$ and speed $=3500 \mathrm{rpm}$ after $1.15 \mathrm{hrs}$ (after $5 \%$ of the life span).

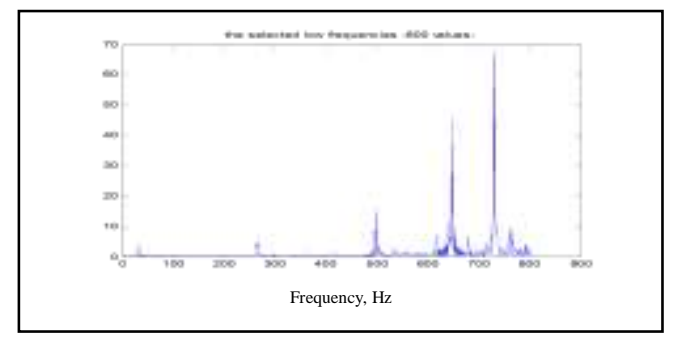

Figure 12-b. DCT analysis of recorded vibration signal at $\mathrm{Load}=1987.5 \mathrm{~N}$ and speed $=3500 \mathrm{rpm}$ after $14.5 \mathrm{hrs}$ (after $60 \%$ of the life span). 
Proc. of the Fifth International Conference on Advances in Mechanical and Robotics Engineering - AMRE 2017. Copyright (C) Institute of Research Engineers and Doctors. All rights reserved. ISBN: 978-1-63248-123-8 doi: 10.15224/ 978-1-63248-123-8-15

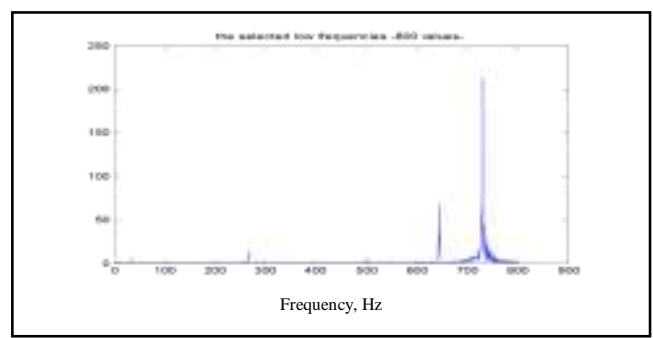

Figure 12-c. DCT analysis of recorded vibration signal at, $\mathrm{Load}=1987.5 \mathrm{~N}$ and speed $=3500 \mathrm{rpm}$ after $20 \mathrm{hrs}$ (after $92 \%$ of the life span).

\section{v. The Neuro Predictor}

Artificial neural networks represent a large class of parallel processing architectures useful in solving several types of problems. The ANN consists of multiple units called neurons or nodes that can perform transformations by means of their state response to input information. A neural network is firstly trained iteratively with a set of samples, known as the training set. [17-19]. Using the training set the artificial neural network can learn the values of its internal parameters [20]. The RUL of test bearings was predicted using an artificial neural network (ANN). Using each of the three described input features, three computer programs have been designed under MATLAB (R14).The programs are used to build, train, optimize the construction of the ANN for the considered groups of inputs and test the precision of prediction. Accurate RUL prediction provides early warning capability where it will be possible to detect, estimate the severity of and isolate faults [21-23].

In the present work three ANNs were designed, trained and validated for the prediction of bearing RUL based on features extracted from the vibration signals. Inputs to the three ANNs included the applied load on the bearing in Newtons, the rotational speed in RPM and the test time along the bearing service life. Besides these three inputs, the first ANN was fed by the time domain vibration signal, the second ANN was fed with the dominant harmonics of the FFT of the vibration signal and the third ANN was fed with 20 selected DCT coefficients of the vibration signal. In all cases the ED estimated from the overall vibration level expressed in RMS values was used as a measure of bearing remaining useful life.

The number of input nodes in the ANN input layer was 8192 in the case of using the vibration values in the time domain, 150 nodes representing the dominant FFT harmonics or 20 selected DCT coefficients. The input vectors at the specified measurement times are input as a matrix with each column representing an input vector. The output layer of the ANN is an identity matrix with diagonal length equal to the number of input vectors. The activation function between input and hidden layers and between hidden and output layer is assumed to be pure linear. The maximum number of epochs for training was taken to be 50000; the error goal was $\left(10^{-6}\right)$.
Three MATLAB (R14) programs were built for training and testing the ANN depending on the nature of and the number of inputs. Based on the extracted features considered, the network was trained using the preliminary parameters of construction. Based on obtained initial results, a final ANN configuration was optimized. Fig. 13 shows a typical training curve for one of the applied ANNs. The desired relative error was reached after 329 epochs (training runs).

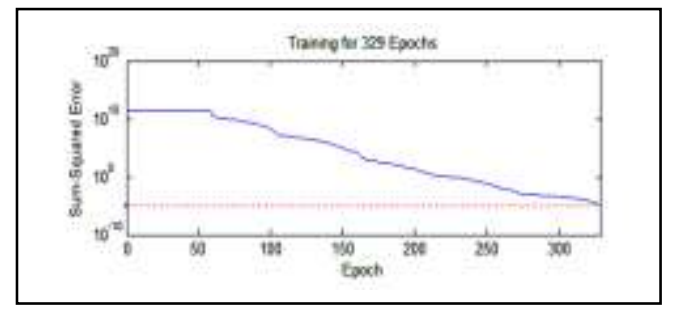

Figure 13. Training epochs and the sum squared error of the input

After training and getting the most suitable network configuration, a test mode was applied to ensure that the network will give the same response when the same inputs are applied at any time. The test results are represented in Table 1.

TABLE 1. Results of the ANN predictor for the case of $\mathrm{P}=1987.5 \mathrm{~N}$ and $\mathrm{S}=3500 \mathrm{rpm}$ Of FV Bearing

\begin{tabular}{|c|c|c|c|}
\hline $\begin{array}{c}\text { Features Extraction } \\
\text { Techniques }\end{array}$ & Time-Domain & FFT & SDCT \\
\hline $\begin{array}{l}\text { No. of trained } \\
\text { samples }\end{array}$ & 8192 & 150 & 20 \\
\hline Max. epochs & 10000 & 10000 & 10000 \\
\hline Error goal & 0.000001 & 0.00001 & 0.000001 \\
\hline Max. learning rate & 0.01 & 0.01 & 0.01 \\
\hline Training epochs & $\begin{array}{c}871,870,1424, \\
1366,1688,676, \\
645,395,345\end{array}$ & $\begin{array}{l}312,313.346, \\
219,354,329, \\
175,492,323\end{array}$ & $\begin{array}{c}111,119,105, \\
79,116,169, \\
95,149,369\end{array}$ \\
\hline No. of I/P neurons & 8192 & 150 & 20 \\
\hline Defined sample & 45 & 45 & 45 \\
\hline No. of tested samples & 45 & 45 & 45 \\
\hline undefined sample & 0 & 0 & 0 \\
\hline No. of hidden neurons & 100 & 100 & 100 \\
\hline Absolute error & $-0.015: 0.015$ & $-0.005: 0.0035$ & $-0.025: 0.005$ \\
\hline Relative error & $\begin{array}{l}-6 * 10^{-4} \\
: 10^{*} 10^{-4}\end{array}$ & $\begin{array}{l}-4 * 10^{-4} \\
: 4 * 10^{-4}\end{array}$ & $\begin{array}{l}-5.5^{*} 10^{-4} \\
: 2.5^{*} 10^{-4}\end{array}$ \\
\hline
\end{tabular}

The results proved that the designed ANNs are all capable of accurate prediction of tested bearing RUL based on the specific features extracted from the vibration signals. A maximum relative error of $\left(-6^{*} 10^{-4}\right.$ to $\left.10^{-3}\right)$ was recorded when using the time domain vibration signal (8192 neurons). On the other hand, a minimum relative error of $\left(-4 * 10^{-4}: 3 * 10^{-4}\right)$ was recorded when using the dominant FFT harmonics of the vibration signal (150 neurons). A relative error of $\left(-5.5^{*} 10^{-4}\right.$ to $2.5^{*} 10^{-4}$ ) was recorded when using the selected DCT coefficients of the vibration signal (20 neurons).

These results indicate a good performance for the proposed neuro RUL predictor with both FFT and DCT. The experiment was repeated for a wider range of load and speed and similar results were confirmed. 


\section{vi. CONCLUSIONS}

A test rig was designed and built to perform accelerated life test of deep groove ball bearings. Effect of bearing load and speed on the bearing life was investigated. Overall vibration level expressed in RMS values of tested bearings was used to evaluate the state of health of the bearings. Euclidean distance (ED) estimated from the overall vibration level expressed in RMS was used to build bearing degradation curve and measure bearing RUL. Spectral analysis of recorded bearing vibration signals along its service life in the form of FFT and DCT was analyzed and related to the state of health of tested bearings.

An ANN neuro-predictor as an artificial intelligence tool has been proposed to achieve accurate estimate of the RUL of a bearing. The proposed ANN model uses three forms of input features. Besides the bearing load, speed, and time along bearing service life, inputs to the ANN included the time domain vibration signal, the dominant FFT harmonics or 20 selected DCT coefficients. The bearing RUL expressed in terms of estimated ED values was taken as the output. A maximum relative error of $\left(-6^{*} 10^{-4}\right.$ to $\left.10^{-3}\right)$ was recorded when using the time domain vibration signal (8192 neurons). A minimum relative error of $\left(-4 * 10^{-4}: 3 * 10^{-4}\right)$ was recorded when using the dominant FFT harmonics of the vibration signal (150 neurons). A relative error of $\left(-5.5^{*} 10^{-4}\right.$ to $\left.2.5^{*} 10^{-4}\right)$ was recorded when using the selected DCT coefficients of the vibration signal (20 neurons).

Obtained results prove that the proposed ANN provides an effective tool for predicting the RUL of bearings using an accelerated life test. Input features in the case of FFT harmonics and DCT coefficients are more practical due to the limited number of input features to the ANN.

\section{REFRENCES}

[1] Warwick Manufacturing Group, "An Introduction to Reliability Engineering", Section 7, University of Warwick, 2007.

[2] A. Heng, S. Zhang, A. C.Tan, J. Mathew, "Rotating machinery prognostics: State of the art, challenges and opportunities", Mechanical Systems and Signal Processing, vol. 23, pp.724-739, 2009.

[3] http://www.weibull.com/basics/lifedata.htm

[4] M. Herzog, "Machine and Component Residual Life Estimation through the Application of Neural Networks". Thesis MD, University of Pretoria, Faculty of engineering, pp. 3-10, 2006.

[5] A. Heng, A.Tan, J. Mathew, N. Montgomery, D. Banjevic, A. Jardine, "Intelligent condition-based prediction of machinery reliability", Mechanical Systems and Signal Processing, 2009.

[6] G. Vachtsevanos, F. Lewis, M. Roemer, A. Hess, B. Wu, "Intelligent Fault Diagnosis and Prognosis for Engineering Systems", John Wiley \& Sons, 2006.

[7] Tushar Salunkhe, N.I. Jamadar, Dr. S. B. Kivade, "Prediction of Remaining Useful Life of Mechanical Components-A Review", International Journal of Engineering Science and Innovative Technology (IJESIT), Vol. 3, Issue 6, November 2014.

[8] J. Sikorska, M.Hodkiewicz, L.Mac, "Prognostic modeling options for remaining useful life estimation by industry", Mechanical Systems and Signal Processing, vol. 25, pp. 1803-1836, 2010.

[9] FSB Bearing Catalogue
[10] H. Liao, W. Zhao, H. Guo, Predicting remaining useful life of an individual unit using proportional Hazards model and logistic regression model, Reliability and Maintainability Symposium, 2006, pp. 127-132.

[11] http://www.econ.upf.edu/ michael/stanford/maeb4.pdf

[12] N. Ahmed, T. Natarajan, K. Rao, "Discrete Cosine Transform", IEEE Transactions On Computers, pp. 90-93, 1974.

[13] 15- R. Desale, S. Verma "Study and Analysis of PCA, DCT \& DWT based Image Fusion Techniques" International Conference on Signal Processing, Image Processing and Pattern Recognition, 2013.

[14] Z.Hafed, M.Levine, "Face Recognition Using The Discrete Cosine Transform", International Journal Of Computer Vision, vol. 43(3), pp. 167-188, 2001.

[15] B. Shekar, G. Thippeswamy, M. Sharmila, "Face Recognition Based on Fractional Discrete Cosine Transform" IEEE-International Conference on Recent Trends in Information Technology, ICRTIT, 2011.

[16] Yujian Qiang, Ling Chen, Liang Hua, Juping Gu, Lijun Ding, Yuqing Liu, Research On The Classification For Faults Of Rolling Bearing Based On Multi-Weights Neural Network, International Journal On Smart Sensing And Intelligent Systems Vol.7, No.3, 2014.

[17] http://www.brighthubpm.com/six-sigma/13445-understandinghistograms-and-their-uses/Histogram

[18] M. Abd Kadir, S. Sharifah, H. Takashi, "Predicting remaining useful life of rotating machinery based artificial neural network", Computers and Mathematics with Applications, vol. 60, pp.1078-1087, 2010.

[19] Z. Tian, L. Wong, N. Safaei, "A neural network approach for remaining useful life prediction utilizing both failure and suspension histories", Mechanical Systems and Signal Processing, vol. 24, pp.1542-1555, 2010.

[20] Taskin Kavzoglu, Determining Optimum Structure for Artificial Neural Networks, 25th Annual Technical Conference, pp. 675-682, 1999.

[21] A.M.Creetu, E.M_Petriu and G.G. "Party-neural network architecture for 3D object representation," IEEE conference 2003.

[22] Andrew K.S. Jardine, Daming Lin and Dragan Banjevic, "A review on machinery diagnostics and prognostics implementing condition-based maintenance", Mechanical Systems and Signal Processing, vol. 20, pp.1483-1510, 2006.

[23] Simon Malinowski, Brigitte Chebel-Morello, Noureddine Zerhouni. Remaining Useful Life estimation based on discriminating shapelet extraction. Reliability Engineering and System Safety, Elsevier, 142, pp.279-288, 2016.

About Author (s):

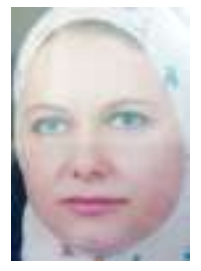

Dalia M. Ammar

Assisstant Lecturer

Production Engineering Dept, Faculty of

Engineering, Pharos University, Egypt.

Fields of interest include: Quality and Reliability.

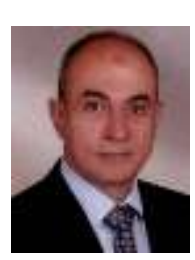

Mohammad A. Younes

Professor of Metrology and Quality

B.Sc. (with hounors), M. Sc, and Ph.D.

Production Engineering Dept, Faculty of

Engineering, Alexandria University, Egypt.

Fields of interest include: Dimensional Metrology,

Nano-Metrology, Quality and Reliability.

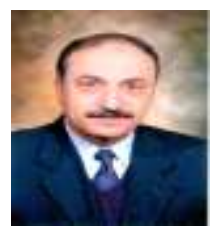

Elsayed S. Elsayed

Assistant Professor

Faculty of Engineering, Alexandria University, Egypt.

Machine tool Dynamics - Condition Monitoring. 